\title{
Effects of red wine flavonoid components on biomembranes and cell proliferation
}

\author{
This article was published in the following Dove Press journal: \\ International Journal of Wine Research \\ 3 May 2011 \\ Number of times this article has been viewed
}

\author{
Hironori Tsuchiya \\ Department of Dental Basic \\ Education, Asahi University School \\ of Dentistry, Mizuho, Gifu, Japan
}

Correspondence: Hironori Tsuchiya Department of Dental Basic Education, Building 3, Asahi University School of Dentistry, I85I-I Hozumi, Mizuho, Gifu 50I-0296, Japan

$\mathrm{Tel}+8 I 58329$ I266

Fax +8I 58329 I266

Email hiro@dent.asahi-u.ac.jp
Background: In order to understand the antiproliferative effect of red wine in mechanistic terms, the membrane interactions of flavonoid components and their related structures were compared using liposomal biomimetic membranes prepared with different phospholipids and cholesterol.

Methods and results: A series of fluorescence polarization measurements revealed that anthocyanidins, flavones, flavonols, flavanones, flavanonols, isoflavones, catechins, and chalcones interact with biomimetic membranes in a structure-dependent manner to decrease their fluidity at concentrations of $1-10 \mu \mathrm{M}$ by preferentially acting in the deeper regions of the lipid bilayers. In the structure and membrane interactivity relationship, greater membraneinteracting potency was associated with a 3-hydroxyl group and a double bond between the 2-carbon and 3-carbon of the $\mathrm{C}$ ring, 3',4'-dihydroxyl groups of the $\mathrm{B}$ ring, and 5,7-dihydroxyl groups of the A ring. Cyanidin, quercetin, and (-)-epigallocatechin gallate meet these structural requirements, and were effective in inhibiting the proliferation of tumor cells, showing inhibition rates of $16.4 \%$ and $35.4 \%, 23.3 \%$ and $74.3 \%$, and $31.3 \%$ and $75.5 \%$, respectively, after culture for 24 and 48 hours. These antiproliferative flavonoids simultaneously decreased the membrane fluidity of tumor cells depending on culture time. The rank order of cell membrane rigidification $[(-)$-epigallocatechin gallate $>$ quercetin $>$ cyanidin] was consistent with inhibition of cell proliferation.

Conclusion: Membrane interaction is very likely to underlie the antiproliferative effects of wine flavonoids. Membrane-interactive flavonoid components would contribute to the functionality of red wine.

Keywords: red wine, flavonoid, membrane interaction, fluidity change, antiproliferative effect

\section{Introduction}

In addition to being one of the most popular alcoholic beverages, red wine is referred to as a functional beverage which potentially exhibits a variety of pharmacological and nutraceutical effects. Its health benefits have been attracting much attention since the term "French paradox" was used to explain the finding that the French population has a relatively low incidence of coronary heart disease despite a high dietary intake of saturated fatty acids, elevated cholesterol levels, and a high rate of cigarette smoking. ${ }^{1}$ Moderate daily consumption of red wine is the most likely explanation for this phenomenon, with an inverse relationship seen between disease risk and red wine intake. Red wine is known to reduce the risk of atherosclerosis, allergy, and cancer, and to lower blood pressure, as well as inhibit lipid oxidation, platelet aggregation, inflammatory reactions, and microbial growth. ${ }^{1-6}$ Most of these properties 
are hypothetically related to a series of wine components with the polyphenol structure (hydroxyl groups on aromatic rings). Substantial quantities of polyphenols are contained in grape skins, seeds, pulps, and musts, and undergo extraction during the wine-making process. ${ }^{7,8}$

Wine polyphenols are composed of two categories, ie, flavonoid and nonflavonoid compounds (stilbenes, phenolic acids, and lignans). Apart from resveratrol, belonging to the stilbene group, the most abundant polyphenols in wine include several classes of hydroxyflavonoids, such as flavonols, flavones, flavanonols, flavanones, isoflavones, isoflavanones, flavanols (or catechins), and anthocyanidins. ${ }^{9}$ These flavonoids share a common structure, consisting of two benzene $\mathrm{A}$ and $\mathrm{B}$ rings bound together by three carbon atoms to form an oxygenated heterocyclic pyran or pyrone $\mathrm{C}$ ring (Figure 1). Their further subdivision is based on the presence or absence of an oxy group at the 4-position, double bond(s) in the $\mathrm{C}$ ring, and a hydroxyl group at the 3-position. ${ }^{10}$ Flavonoids have a phenyl group at the 2-position, whereas isoflavonoids have this group at the 3-position. The structural diversity depends on the number of hydroxyl groups in the $\mathrm{A}$ and $\mathrm{B}$ rings. Chalcones and dihydrochalcones are also flavonoids, but lack a $\mathrm{C}$ ring. ${ }^{10}$

In addition to their influence on the quality, color, and taste of wine, ${ }^{7}$ flavonoid components are possibly responsible for the antioxidant, antithrombotic, anti-inflammatory, antiallergic, antimicrobial, antiviral, apoptosis-inducing, anticarcinogenic, antitumor, and antiproliferative properties of red wine, as well as nonflavonoid components. ${ }^{11}$ They are best known for their antioxidant and antiproliferative effects. Both effects have been explained by the prevention of lipid peroxidation, the scavenging of reactive oxygen species, and the inhibition or modification of radical generation-associated and cell proliferation-associated enzymes (eg, cyclo-oxygenase, phospholipase, lipoxygenase, ornithine decarboxylase, tyrosine-specific protein kinase, and mitogen-activated protein kinase), receptors (eg, the estrogen binding site), channels, transporters, and signal transduction systems. ${ }^{10,12}$ These mechanistically relevant events occur in the lipid membrane environment, within and through the lipid bilayers. The ability to access membrane-embedded enzymes, receptors, and channels also involves interaction with membrane lipids. Although the red wine flavonoids are all structurally different, a common mode of action in biomembranes is presumed.

The bioactivity of the flavonoids is linked with their ability to interact with biological membranes. ${ }^{13,14}$ Interaction with membrane lipid bilayers to change their physicochemical properties is one of the pharmacological mechanisms used by antioxidant, antimicrobial, antithrombotic, anti-inflammatory, and antiproliferative drugs and phytochemicals. ${ }^{15-20}$

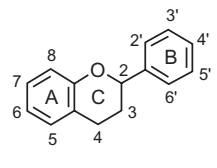

Flavonoids

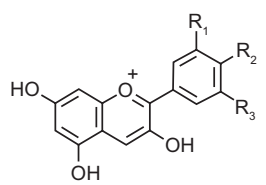

Anthocyanidins

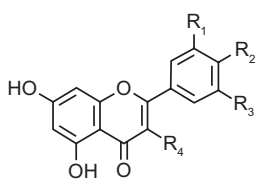

Flavones and Flavonols

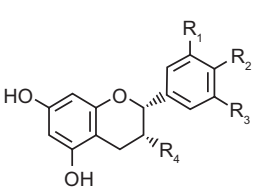

Flavanols or Catechins

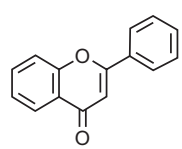

Flavone

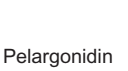

Cyanidin

Delphinidin $\mathrm{OH} \quad \mathrm{OH} \quad \mathrm{OH}$

$\begin{array}{lllll} & \mathrm{R}_{1} & \mathrm{R}_{2} & \mathrm{R}_{3} & \mathrm{R}_{4} \\ \text { Apigenin } & \mathrm{H} & \mathrm{OH} & \mathrm{H} & \mathrm{H} \\ \text { Luteolin } & \mathrm{OH} & \mathrm{OH} & \mathrm{H} & \mathrm{H} \\ \text { Kaempferol } & \mathrm{H} & \mathrm{OH} & \mathrm{H} & \mathrm{OH} \\ \text { Quercetin } & \mathrm{OH} & \mathrm{OH} & \mathrm{H} & \mathrm{OH} \\ \text { Myricetin } & \mathrm{OH} & \mathrm{OH} & \mathrm{OH} & \mathrm{OH}\end{array}$

(-)-Epicatechin

(-)-Epigallocatechin

(-)-Epigallocatechin gallate $\mathrm{OH}$

$\mathrm{R}_{1} \quad \mathrm{R}_{2}$

$\begin{array}{llll}\mathrm{OH} & \mathrm{OH} & \mathrm{H} & \mathrm{OH}\end{array}$

$\mathrm{OH} \mathrm{OH} \mathrm{OH} \mathrm{OH}$

$\mathrm{OH}$

$\mathrm{OH}$

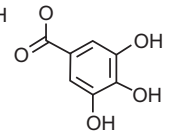

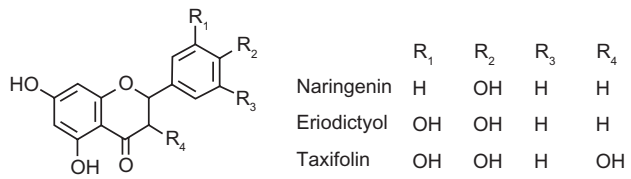

Flavanones and Flavanonol

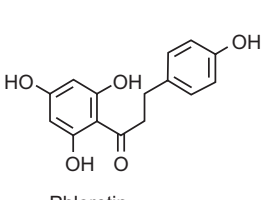

Phloretin

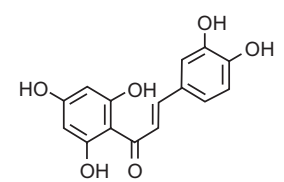

2',4',6',3,4-Pentahydroxychalcone
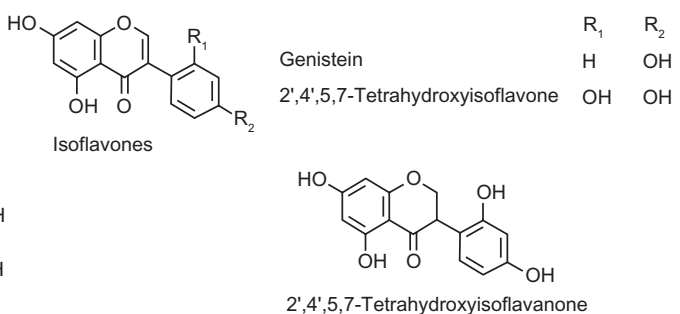

Figure I Wine flavonoids and related structures tested in this study. 
The objective of this study was to compare the interactions of flavonoids and their related structures with biomembranes in order to understand the beneficial effects of red wine from the viewpoint of mechanistic membrane interaction. The flavonoids tested were anthocyanidins (pelargonidin, cyanidin, and delphinidin), flavones (apigenin and luteolin), flavonols (kaempferol, quercetin, and myricetin), flavanones (naringenin and eriodictyol), flavanonol (taxifolin), catechins [(-)-epicatechin, $(-)$-epigallocatechin and (-)-epigallocatechin gallate], chalcones (phloretin and 2', $4^{\prime}, 6^{\prime}, 3,4$-pentahydroxychalcone), isoflavones (genistein and 2',4',5,7-tetrahydroxyisoflavone), isoflavanone (2',4',5,7-tetrahydroxyisoflavanone), and parent structures without hydroxyl groups (Figure 1). Their potencies in interaction with biomimetic and cellular membranes to modify fluidity were determined by measuring fluorescence polarization using a series of fluorescent probes localized differently in the lipid bilayers. Based on the comparative results, tumor cell cultures were exposed to the most membraneinteractive flavonoids to investigate the antiproliferative effects associated with their membrane interaction.

\section{Materials and methods Chemicals}

Quercetin, (-)-epicatechin, (-)-epigallocatechin, and (-)-epigallocatechin gallate were supplied by Professor Toshiyuki Tanaka of Gifu Pharmaceutical University, Gifu, Japan. Flavonoids other than quercetin and catechins were purchased from Funakoshi (Tokyo, Japan). 1-Palmitoyl-2-oleoylphosphatidylcholine (POPC), 1-palmitoyl-2-oleoylphosphatidylethanolamine (POPE), and 1-stearoyl-2-oleoylphosphatidylserine (SOPS) were obtained from Avanti Polar Lipids (Alabaster, AL), and cholesterol from Wako Pure Chemicals (Osaka, Japan). 2-(9Anthroyloxy)stearic acid (2-AS), 6-(9-anthroyloxy)stearic acid (6-AS), 9-(9-anthroyloxy)stearic acid (9-AS), 12-(9anthroyloxy)stearic acid (12-AS), and 16-(9-anthroyloxy) palmitic acid (16-AP) were purchased from Molecular Probes (Eugene, OR). The dimethyl sulfoxide and acetone used for preparing the reagent solutions were of spectroscopic grade (Kishida, Osaka, Japan), and both the water and ethanol were of liquid chromatographic grade (Kishida). Dulbecco's phosphate-buffered saline of pH 7.4 was obtained from Dainippon Pharmaceuticals (Osaka, Japan). All other chemicals were of the highest analytical grade available.

\section{Preparation of biomimetic membranes}

In a previous membrane interaction study of flavonoids, ${ }^{21}$ liposomes were prepared by sonicating lipid dry films in buffer with a microtip made of metal. However, the flavonoids were reported to chelate metal ions between 5-hydroxyl and 4-oxo groups, at ortho-dihydroxyl groups of the $\mathrm{B}$ ring, and at a 3-hydroxyl group of the $\mathrm{C}$ ring, resulting in oxidative degradation. ${ }^{22,23}$ Such chelation would lead to an underestimation of the inherent membrane effects of flavonoids. Therefore, in this study, liposomal biomimetic membranes were prepared by the injection method without using a metal microtip as reported previously, ${ }^{24}$ with some modifications as follows. An aliquot $(250 \mu \mathrm{L})$ of POPC, POPE, SOPS, and cholesterol (48:24:8:20, mol\%; total lipids $10 \mathrm{mM}$ ) in ethanol solution was injected four times into $199 \mathrm{~mL}$ of phosphate-buffered saline under stirring above the phase-transition temperatures of phospholipids. The molar ratio of membrane lipids was adjusted to the composition of the major membrane lipids in tumor cells. ${ }^{20}$

\section{Determination of membrane interactivity}

The membrane interactions of flavonoids were compared by determining the potency of their ability to modify the fluidity of biomimetic membranes. An aliquot of flavonoid in dimethyl sulfoxide solution was added to the membrane preparations to give a final concentration of $1 \mu \mathrm{M}$ and $10 \mu \mathrm{M}$ for each flavonoid, followed by incubation at $37^{\circ} \mathrm{C}$ for 30 minutes. The dimethyl sulfoxide concentration was less than $0.125 \%(\mathrm{v} / \mathrm{v})$ of the total volume, so as not to affect membrane fluidity. Thereafter, the membranes were labeled with 2-AS, 6-AS, 9-AS, 12-AS, or 16-AP by incubating with an aliquot of probe solution in acetone at $37^{\circ} \mathrm{C}$ for 1.5 hours. ${ }^{25}$ The molar ratio of $n-\mathrm{AS}(\mathrm{P})$ to total membrane lipids was 1:210 and the acetone concentration was $0.125 \%(\mathrm{v} / \mathrm{v})$ of the total volume. Because flavonoids could interfere with fluorescence polarization measurements at submillimolar levels due to their fluorescence-quenching properties ${ }^{26}$ concentrations of $10 \mu \mathrm{M}$ or less were used in this study. Fluorescence polarization was measured at excitation and emission wavelengths of $367 \mathrm{~nm}$ and $443 \mathrm{~nm}$, respectively, by an RF-540 spectrofluorometer (Shimadzu, Kyoto, Japan) equipped with a polarizer and a cuvette thermocontrolled at $37^{\circ} \mathrm{C}$. An increase in fluorescence polarization is indicative of a decrease in membrane fluidity (membrane rigidification). Fluorescent probes, $n$-AS(P), selectively locate at a graded series of levels in lipid membranes. ${ }^{25}$ The $n$-AS(P) polarization values decrease with increasing $n$ because the deeper regions of the lipid bilayers are more fluid than the superficial regions. Therefore, the comparative effects of flavonoids at different membrane depths were evaluated by the $n$ - $\mathrm{AS}(\mathrm{P})$ polarization changes (\%) relative to control polarization values. 


\section{Antiproliferative activity analysis}

The effects of membrane-interactive flavonoids on cell proliferation were analyzed as reported previously. ${ }^{20}$ Briefly, mouse myeloma cells (Sp2/O-Ag14) were inoculated at $2.0 \times 10^{5}$ cell $/ \mathrm{mL}$ in Dulbecco's modified Eagle's medium supplemented with $10 \%$ (v/v) fetal calf serum (ICN Biomedicals, Aurora, $\mathrm{OH})$. This cell line was chosen because of its suitability for membrane fluidity experiments in which cells were successfully suspended in phosphate-buffered saline and labeled with fluorescent probes. Aliquots of cyanidin, quercetin, and (-)-epigallocatechin gallate in dimethyl sulfoxide solution were added to the culture medium to give a final concentration of $10 \mu \mathrm{M}$ for each. The final concentration of dimethyl sulfoxide was $0.5 \%(\mathrm{v} / \mathrm{v})$ so as not to affect cell proliferation. After culture at $37^{\circ} \mathrm{C}$ for 24 and 48 hours in a humidified $5 \%$ $\mathrm{CO}_{2}$ atmosphere, the number of viable (trypan blue stainingnegative) cells was counted by a hemocytometer, followed by comparison with controls (treated with dimethyl sulfoxide vehicle) to determine cell viability (\%).

\section{Effects on cell membranes}

Mouse myeloma cells were cultured with and without cyanidin, quercetin, and (-)-epigallocatechin gallate $(10 \mu \mathrm{M}$ for each) as described in the above method for antiproliferative activity analysis. A $2 \mathrm{~mL}$ aliquot of cell culture was obtained after culture for 24 and 48 hours, followed by centrifugation to collect the cells, which were washed twice and suspended in phosphate-buffered saline. The cells were labeled with 2-AS and 12-AS, and the effects of flavonoids on the cell membranes were then determined by measuring fluorescence polarization as described in the method for determination of membrane interaction. The 2-AS and 12-AS polarization changes (\%) relative to control polarization values were used to evaluate the effects of flavonoids on cell membranes and their sites of action in the membrane lipid bilayers.

\section{Statistical analysis}

All results are expressed as mean \pm standard error of the mean ( $\mathrm{n}=7$ for membrane fluidity experiments and $\mathrm{n}=5$ for cell culture experiments). Data were statistically analyzed by one-way analysis of variance, followed by post hoc Fisher's protected least significant difference test using StatView (v 5.0; SAS Institute, Cary, NC). $P$ values $<0.05$ were considered to be statistically significant.

\section{Results}

\section{Interactions with biomimetic membranes}

The flavonoids interacted with the biomimetic membranes to increase $n-\mathrm{AS}(\mathrm{P})$ polarization at $1-10 \mu \mathrm{M}$, indicating that they decreased membrane fluidity in a structurally dependent manner by acting at different regions of the lipid bilayers (Table 1). Their membrane-rigidifying effects were significantly influenced by the presence, number, and position of the hydroxyl groups. Anthocyanidins rigidified the biomimetic membranes, with the potency being delphinidin $<$ pelargonidin $<$ cyanidin. In particular, delphinidin aggregated the liposomes at $10 \mu \mathrm{M}$. Aggregation of liposomes is inducible by the highly polar flavonoids, to which delphinidin belongs, because of five hydroxyl groups and an oxonium ion in its $\mathrm{C}$ ring. ${ }^{27}$ Flavanones, flavones, and flavonols interacted with biomimetic membranes in an increasing order of intensity, ie, naringenin $<$ apigenin $<$ kaempferol, and eriodictyol $<$ luteolin $<$ quercetin. Quercetin was the most active of the flavonols, followed by kaempferol and myricetin. Quercetin, a flavonol, was also more active than taxifolin, the structurally corresponding flavanonol. As shown by a comparison between apigenin and genistein, flavonoids were more effective in rigidifying the membranes than isoflavonoids. For the isoflavonoids, the rank order of membrane interactivity was $2^{\prime}, 4^{\prime}, 5,7$-tetrahydroxyisoflavanone $<$ genistein $\left(4^{\prime}, 5,7\right.$-trihydroxyisoflavone $)<2^{\prime}, 4^{\prime}, 5,7-$ tetrahydroxyisoflavone. Chalcones and catechins showed membrane-rigidifying potency being a parent structure (chalcone) $<2^{\prime}, 4^{\prime}, 6^{\prime}, 3,4$-pentahydroxychalcone $<$ phloretin, and being $(-)$-epigallocatechin $=(-)$-epicatechin $<$ (-)-epigallocatechin gallate. When comparing the related structures at an equimolar concentration of $10 \mu \mathrm{M}$, cyanidin, quercetin, and (-)-epigallocatechin gallate had the highest membrane interaction for each flavonoid subclass.

Many flavonoids tended to increase the relative changes in $n$-AS(P) polarization with increasing $n$ (Table 1 ), suggesting that they were effective at the deeper regions of the membrane lipid bilayers rather than at the superficial regions. Cyanidin, quercetin, and (-)-epigallocatechin gallate showed the largest increase in 16-AP polarization compared with $n$-AS polarization. The relative ratios of 16-AP polarization increases to 2-AS polarization increases at $10 \mu \mathrm{M}$ were 2.65 for cyanidin, 3.02 for quercetin, and 3.36 for (-)-epigallocatechin gallate.

\section{Effects on cell proliferation}

The effects of membrane-interactive flavonoids ( $10 \mu \mathrm{M}$ for each) on cell viability are shown in Figure 2. The mean inhibition produced by cyanidin, quercetin, and (-)-epigallocatechin gallate was $16.4 \%, 23.3 \%$, and $31.3 \%$, respectively, after culture for 24 hours, and $35.4 \%, 74.3 \%$, and $75.5 \%$, respectively, 
Table I Effects of flavonoids on fluorescence polarization of biomimetic membranes

\begin{tabular}{|c|c|c|c|c|c|}
\hline & \multicolumn{5}{|c|}{ n-AS(P) polarization change $(\%)$ relative to control polarization value } \\
\hline & 2-AS & 6-AS & 9-AS & I2-AS & 16-AP \\
\hline I $\mu \mathrm{M}$ Pelargonidin & $2.78 \pm 0.44^{* *}$ & $2.93 \pm 0.33^{* *}$ & $4.10 \pm 0.47^{* *}$ & $5.72 \pm 0.52^{* *}$ & $7.46 \pm 0.94 * *$ \\
\hline $10 \mu \mathrm{M}$ Pelargonidin & $26.5 \pm 0.43^{* *}$ & $26.2 \pm 0.53^{* *}$ & $38.9 \pm 0.40 * *$ & $59.1 \pm 1.39 * *$ & $63.6 \pm 1.58 * *$ \\
\hline I $\mu \mathrm{M}$ Cyanidin & $4.37 \pm 0.38^{* *}$ & $5.90 \pm 0.4 I^{* *}$ & $5.78 \pm 0.76 * *$ & $8.33 \pm 1.05^{* *}$ & $9.69 \pm 0.08 * *$ \\
\hline I0 $\mu$ M Cyanidin & $40.7 \pm 0.75^{* *}$ & $40.3 \pm 0.5 I^{* *}$ & $54.9 \pm 0.59 * *$ & $84.6 \pm 0.90 * *$ & $108 \pm 1.35^{* *}$ \\
\hline I «M Delphinidin & $3.08 \pm 0.49 * *$ & $2.80 \pm 0.5 I^{* *}$ & $3.18 \pm 0.45^{* *}$ & $4.50 \pm 0.7 I^{* *}$ & $4.22 \pm 1.67^{*}$ \\
\hline $10 \mu \mathrm{M}$ Delphinidin & Aggregated & Aggregated & Aggregated & Aggregated & Aggregated \\
\hline I $\mu$ M Kaempferol & $1.95 \pm 0.30 * *$ & $2.36 \pm 0.33^{* *}$ & $4.11 \pm 0.54^{* *}$ & $6.40 \pm 0.62^{* *}$ & $7.51 \pm\left. 1.3\right|^{* *}$ \\
\hline $10 \mu \mathrm{M}$ Kaempferol & $22.2 \pm 0.47^{* *}$ & $29.5 \pm 0.07^{* *}$ & $39.9 \pm 0.40 * *$ & $59.8 \pm 1.02 * *$ & $57.7 \pm 2.47 * *$ \\
\hline I $\mu \mathrm{M}$ Quercetin & $2.95 \pm 0.22^{* *}$ & $3.86 \pm 0.45 * *$ & $4.98 \pm 0.59 * *$ & $8.83 \pm 0.93 * *$ & $8.02 \pm 1.52^{* *}$ \\
\hline $10 \mu \mathrm{M}$ Quercetin & $26.7 \pm 0.43^{* *}$ & $32.8 \pm 0.46^{* *}$ & $44.8 \pm 0.79 * *$ & $71.6 \pm 0.75^{* *}$ & $80.7 \pm 1.4 I^{* *}$ \\
\hline I $\mu$ M Myricetin & $1.95 \pm 0.42^{* *}$ & $1.73 \pm 0.32 *$ & $1.58 \pm 0.48^{*}$ & $2.36 \pm 0.58^{*}$ & $1.69 \pm 1.16$ \\
\hline $10 \mu \mathrm{M}$ Myricetin & $14.9 \pm 0.38 * *$ & $13.8 \pm 0.37^{* *}$ & $15.2 \pm 0.58 * *$ & $21.5 \pm 1.06 * *$ & $16.5 \pm 1.60 * *$ \\
\hline $10 \mu \mathrm{M}$ Apigenin & $6.98 \pm 0.44 * *$ & $10.1 \pm 0.54^{* *}$ & $12.5 \pm 0.49 * *$ & $13.9 \pm 0.76 * *$ & $22.6 \pm 1.30$ *** \\
\hline $10 \mu \mathrm{M}$ Luteolin & $\mid I .1 \pm 0.49 * *$ & $19.0 \pm 0.24 * *$ & $29.7 \pm 0.34 * *$ & $31.5 \pm 0.58 * *$ & $39.8 \pm 1.17^{* *}$ \\
\hline $10 \mu \mathrm{M}(-)$-Epicatechin & $0.64 \pm 0.57$ & $1.75 \pm 0.53^{*}$ & $3.20 \pm 0.53^{* *}$ & $4.73 \pm 1.02 * *$ & $6.31 \pm 0.69 *$ \\
\hline $10 \mu \mathrm{M}(-)$-Epigallocatechin & $0.55 \pm 0.39$ & $1.65 \pm 0.54^{*}$ & $2.96 \pm 0.59 * *$ & $4.44 \pm 0.34 * *$ & $3.57 \pm 1.66 *$ \\
\hline \multicolumn{6}{|l|}{$10 \mu \mathrm{M}(-)$-Epigallocatechin } \\
\hline gallate & $28.9 \pm 0.69 * *$ & $26.6 \pm 0.18^{* *}$ & $28.1 \pm 0.42^{* *}$ & $58.5 \pm 1.66 * *$ & $97.0 \pm 2.16^{* *}$ \\
\hline $10 \mu \mathrm{M}$ Naringenin & $1.22 \pm 0.35$ & $3.34 \pm 0.23 * *$ & $3.58 \pm 0.86 * *$ & $5.02 \pm 0.7 I^{* *}$ & $4.19 \pm 2.05$ \\
\hline $10 \mu \mathrm{M}$ Eriodictyol & $5.08 \pm 0.34 * *$ & $5.58 \pm 0.28^{* *}$ & $7.13 \pm 0.43^{* *}$ & $11.3 \pm 0.89 * *$ & $8.69 \pm 1.89 * *$ \\
\hline $10 \mu \mathrm{M}$ Taxifolin & $3.89 \pm 0.4 I^{* *}$ & $5.90 \pm 0.69 * *$ & $6.00 \pm 0.45^{* *}$ & $8.14 \pm 0.53^{* *}$ & $9.11 \pm 2.17^{* *}$ \\
\hline $10 \mu \mathrm{M}$ Phloretin & $23.1 \pm 0.45^{* *}$ & $22.2 \pm 0.49 * *$ & $23.0 \pm 0.69 * *$ & $34.7 \pm 0.70 * *$ & $23.1 \pm 1.36 * *$ \\
\hline \multicolumn{6}{|l|}{$10 \mu \mathrm{M} 2^{\prime}, 4^{\prime}, 6^{\prime}, 3,4-$} \\
\hline Pentahydroxychalcone & $8.24 \pm 0.29 * *$ & $10.4 \pm 0.29 * *$ & $11.9 \pm 0.44 * *$ & $22.1 \pm 0.59 * *$ & $10.6 \pm\left. 1.4\right|^{* *}$ \\
\hline $10 \mu \mathrm{M}$ Genistein & $1.83 \pm 0.46^{* *}$ & $2.51 \pm 0.50 * *$ & $3.77 \pm 0.52^{* *}$ & $6.25 \pm 0.85^{* *}$ & $6.98 \pm 2.67^{*}$ \\
\hline \multicolumn{6}{|l|}{$10 \mu \mathrm{M}$ 2',4',5,7- } \\
\hline Tetrahydroxyisoflavone & $5.86 \pm 0.50 * *$ & $5.90 \pm 0.50 * *$ & $6.77 \pm 0.34 * *$ & $8.83 \pm 0.73 * *$ & $7.59 \pm 1.69 * *$ \\
\hline \multicolumn{6}{|l|}{$10 \mu \mathrm{M} 2,4^{\prime}, 5,7-$} \\
\hline Tetrahydroxyisoflavanone & $0.70 \pm 0.54$ & $2.18 \pm 0.50^{* *}$ & $3.47 \pm 0.74 * *$ & $4.55 \pm 0.6 I^{* *}$ & $5.61 \pm 1.80^{*}$ \\
\hline $10 \mu \mathrm{M}$ Flavone & $0.90 \pm 0.41$ & $0.70 \pm 0.64$ & $1.29 \pm 0.93$ & $0.93 \pm 0.51$ & $1.59 \pm 1.96$ \\
\hline $10 \mu \mathrm{M}$ Flavanone & $0.15 \pm 0.45$ & $0.34 \pm 0.32$ & $0.67 \pm 0.61$ & $0.53 \pm 1.01$ & $1.31 \pm 2.01$ \\
\hline $10 \mu \mathrm{M}$ Chalcone & $0.73 \pm 0.62$ & $0.68 \pm 0.39$ & $1.47 \pm 0.56$ & $1.16 \pm 0.68$ & $1.36 \pm 1.97$ \\
\hline
\end{tabular}

Notes: Results are expressed as mean \pm standard error of the mean $(n=7) . * P<0.05$ and $* * P<0.01$ versus control.

Abbreviations: 2-AS, 2-(9-anthroyloxy)stearic acid; 6-AS, 6-(9-anthroyloxy)stearic acid; 9-AS, 9-(9-anthroyloxy)stearic acid; 12-AS, 12-(9-anthroyloxy)stearic acid; 16-AP, 16-(9-anthroyloxy)palmitic acid.

after culture for 48 hours. The rank order of antiproliferative activity [(-)-epigallocatechin gallate $>$ quercetin $>$ cyanidin] was not necessarily correlated with that of biomimetic membrane interactivity [cyanidin $>$ quercetin $>(-)$-epigallocatechin gallate].

\section{Effects on cell membranes}

Together with inhibiting cell proliferation, cyanidin, quercetin, and (-)-epigallocatechin gallate rigidified cell membranes, as shown by the 2-AS and 12 -AS polarization increases in Figure 3. Comparisons between 2-AS and 12-AS polarization changes indicated that these flavonoids were more effective at the deeper hydrophobic regions of the cell membranes. The rigidifying effect on cell membranes was more evident in (-)-epigallocatechin gallate after 48 hours of culture time. The relative potency of cell membrane rigidification [(-)-epigallocatechin gallate $>$ quercetin $>$ cyanidin] agreed with that of cell proliferation inhibition $[(-)$-epigallocatechin gallate $>$ quercetin $>$ cyanidin]. 


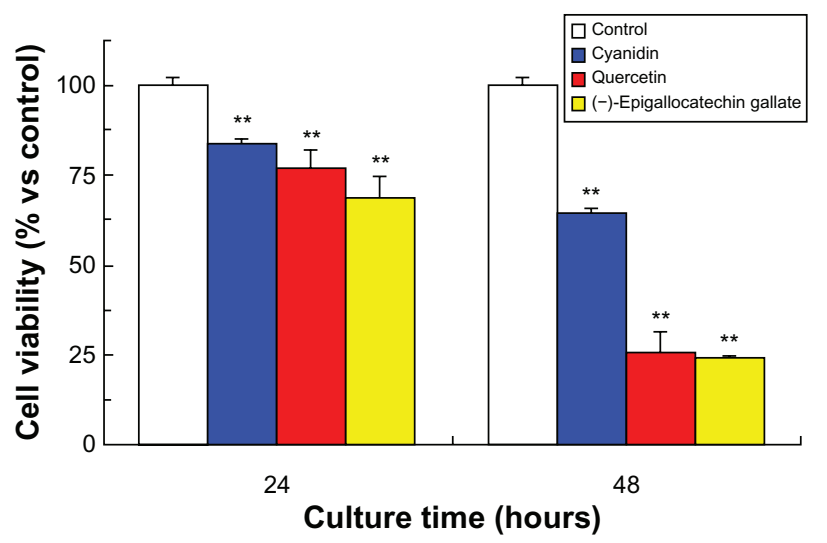

Figure 2 Effects of membrane-interactive flavonoids on cell viability. After tumor cells were cultured with cyanidin, quercetin, and (-)-epigallocatechin gallate (10 $\mu \mathrm{M}$ for each) for 24 and 48 hours, cell viability was determined by a trypan blue dye exclusion method. Results are expressed as mean \pm standard error of the mean $(n=5)$.

Note: $* * p<0.01$ versus control.

\section{Discussion}

The lipid peroxidation-inhibiting, radical-scavenging, and metal-chelating effects of the flavonoids as antioxidants have been widely studied. ${ }^{18,23,28}$ However, inhibition of cell proliferation, apoptosis induction, and tumor-relevant enzyme modification are included in the antitumor mechanisms of flavonoids. The activation and suppression of cell proliferation, apoptosis, and enzymes occur in the lipid membrane environment, and these events are governed by the physicochemical properties of biological membranes..$^{29-31}$ Flavonoids, as well as membrane-acting drugs and phytochemicals, are

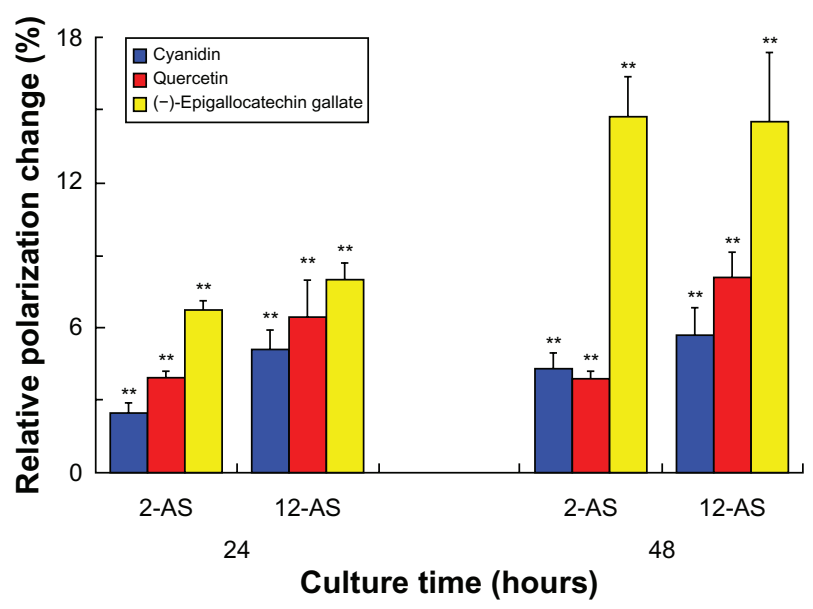

Figure 3 Effects of antiproliferative flavonoids on tumor cell membranes. After tumor cells were cultured with cyanidin, quercetin, and (-)-epigallocatechin gallate (10 $\mu \mathrm{M}$ for each) for 24 and 48 hours, the 2-AS and I2-AS polarization changes (\%) relative to control polarization values were determined.

Notes: Results are expressed as mean \pm standard error of the mean $(n=5)$. $* * P<0.0$ I versus control.

Abbreviations: 2-AS, 2-(9-anthroyloxy)stearic acid; 12-AS, 12-(9-anthroyloxy)stearic acid. assumed to interact with lipid bilayers and modify membrane fluidity. ${ }^{20,32}$ Such membrane interaction is hypothesized to be one of the determinants of the pharmacological and nutraceutical effects of flavonoids. ${ }^{19,21,33}$

Anthocyanidins that are well distributed in the edible parts of plants are cyanidin, pelargonidin, and delphinidin. ${ }^{34}$ The major grape flavonol is quercetin, followed by myricetin and kaempferol. ${ }^{1}$ Substantial concentrations of catechins are also found in red wine. ${ }^{35}$ In this study, the membrane interactivity and antiproliferative activity of these flavonoids and their related structures were compared. Other anthocyanidins (eg, malvidin) and other flavonols (eg, tamarixetin) are also known to be bioactive, ${ }^{36,37}$ but show less activity and have lower concentrations in wine compared with cyanidin and quercetin. ${ }^{34,38}$ Because structural analogs with hydroxyl group(s) at the 3'-, 4'-, and 5'-position of the B ring (Figure 1) were compared in each flavonoid subclass to address the structure and membrane interactivity relationship, the methoxyl flavonoids, ie, malvidin (3',5'-dimethylated delphinidin) and tamarixetin ( $4^{\prime}$-methylated quercetin) were not used in this study. Although wine flavonoids are present as both aglycones and glycosides, the membrane interactions of the glucoside and rutinoside were reported to be much less or negligible compared with their aglycones. ${ }^{21}$ Aglycone flavonols and flavones are more bioactive than their glycosides, while glycosides undergo in vivo hydrolysis to aglycones and increase their bioactivity significantly. ${ }^{39,40}$ Therefore, the present comparisons were focused on flavonoid aglycones. The membranes tested were prepared to resemble the membrane composition and properties of flavonoids targeting tumor cells. ${ }^{20}$ Consequently, wine flavonoids have been shown to interact differently with biomimetic membranes and decrease their fluidity in a structure-dependent manner.

Their amphiphilic properties allow flavonoids to interact not only hydrophobically with phospholipid acyl chains but also electrostatically with phospholipid polar heads. ${ }^{13}$ In addition to hydrophobic interaction and hydrogen bonding, steric configuration also participates in the interaction between flavonoids and membranes. ${ }^{41} \mathrm{~A}$ set of $n$-AS(P) polarization values reflecting the gradient of fluidity from the surface to the center of the lipid bilayers has suggested that membrane-interactive flavonoids preferentially act at the deeper membrane regions by intercalating between the hydrocarbon chains of the biomimetic membranes.

The presence of polyhydroxyl groups, the heterocyclic $\mathrm{C}$ ring (pyran or pyrone), and structural hydrophobicity are important for flavonoids to interact with biomimetic membranes. However, too high or too low hydrophobicity 
hampers the bioactivities of flavonoids. ${ }^{19}$ Comparisons of structurally corresponding flavonoids indicate that a hydroxyl group at the 3 -position of the $\mathrm{C}$ ring induces greater membrane rigidification, as shown by the relative potency of kaempferol $>$ apigenin, and quercetin $>$ luteolin. A double bond between the 2- and 3-carbon of the $\mathrm{C}$ ring is another determinant, as shown by the relative potency being apigenin $>$ naringenin, luteolin $>$ eriodictyol, and quercetin $>$ taxifolin. In flavonols and anthocyanidins, the 3'- and 4'-dihydroxyl groups of their B ring form an ortho-diphenolic (catechol) structure, increasing the membrane effects, as shown by the relative potency of quercetin $>$ kaempferol $>$ myricetin, and cyanidin $>$ pelargonidin $>$ delphinidin. The lower membrane interactivities of myricetin and delphinidin are attributable to their hydrophobicity being reduced by a trihydroxylated $\mathrm{B}$ ring. While a 3-hydroxyl group of the $\mathrm{C}$ ring contributes to enhancing bioactivity by the electron-donating effects of the 5,7-dihydroxyl groups of the A ring, a hydroxyl group on the $\mathrm{B}$ ring also contributes via the electron-donating effect of another hydroxyl group in the catechol moiety. ${ }^{42}(-)$-Epigallocatechin gallate is the most active of the catechins, because of its more hydrophobic and extremely polyhydroxylated structure. ${ }^{14}$ Quercetin molecules penetrate into the hydrophobic regions of lipid bilayers and the boundaries between polar and hydrophobic regions to interact with phospholipid acyl chains. ${ }^{33} 2^{\prime}, 4^{\prime}, 6^{\prime}, 3,4$-Pentahydroxychalcone with its long axis structure shows a relatively weak membrane-rigidifying effect compared with luteolin which has a tricyclic $\left(\mathrm{C}_{6}-\mathrm{C}_{3}-\mathrm{C}_{6}\right)$ skeleton. This may be due to the characteristic intercalation of a chalcone molecule between the polar heads of the membrane phospholipids to produce an opposite effect, ie, membrane fluidization by increasing the distance between phospholipid acyl chains and membrane rigidification by causing interdigitation of lipid bilayers. ${ }^{33}$ Comparisons of corresponding structures indicate that flavonoids are more active than isoflavonoids. The relationship characterized between structure and membrane interactivity is consistent with the structural requirement for flavonoids to have greater bioactivity. ${ }^{10}$

Cyanidin, quercetin, and (-)-epigallocatechin gallate meet the structure and membrane interactivity relationship requirements, and have been shown not only to inhibit the proliferation of tumor cells, but also to decrease the fluidity of tumor cell membranes. The rank order of antiproliferation is (-)-epigallocatechin gallate $>$ quercetin $>$ cyanidin, which is consistent with that of cell membrane rigidification, but not with that of biomimetic membrane rigidification. Anthocyanidins are degraded in culture medium, with half-lives of 30-60 minutes. ${ }^{43}$ Such limited stability of the anthocyanidins may produce the discrepancy seen between biomimetic and tumor cell membranes.

The proliferative abilities of tumor cells are closely associated with the altered physicochemical properties of cell membranes. Neoplastic and metastatic cells have more fluid membranes than their normal counterparts, resulting from increased phospholipid unsaturation degree and decreased cholesterol content. ${ }^{44}$ Membrane rigidification by flavonoids could counteract the increased membrane fluidity of tumor cells.

While antioxidant and anticarcinogenic effects are interrelated in the prevention of disease, flavonoids are also able to inhibit tumor cell proliferation by affecting several key events, ie, inhibition of tumorigenesis-related enzymes, induction of apoptosis, modulation of proliferative signal transduction, arrest of cell cycle progression, and alteration of receptor function. ${ }^{10,12,45-47}$ In the last few decades, cell membranes and membranous organelles have been identified as novel targets for antitumor agents. ${ }^{20,21,48}$

Cyclo-oxygenase, especially the inducible form, cyclooxygenase-2, plays an important pathological role in tumorigenesis as well as in inflammation. ${ }^{49}$ Membrane-interactive (-)-epigallocatechin gallate, quercetin, and cyanidin inhibit cyclo-oxygenase more intensively than the structurally-related flavonoids, affecting tumor cell proliferation. ${ }^{45,46}$ Inhibition of cyclo-oxygenase- 2 expression also depends on the presence of a catechol structure on the flavonoid $\mathrm{B}$ ring, ${ }^{50}$ consistent with the structure and membrane interactivity relationship characterized in this study. The ability to modify membrane fluidity determines the bioactivity associated with cyclo-oxygenase inhibition. ${ }^{51}$ Membrane fluidity changes induced by flavonoids should affect tumorigenesis-relevant enzymes by disturbing the membrane environment optimal for the conformation of enzyme proteins. The apoptotic pathway involves death receptor ligands, eg, tumor necrosis factor alpha, which are bound to the membrane receptor, eg, the tumor necrosis factor receptor. Activation of this receptor leads to the activation of caspase and mitogen-activated protein kinase, ultimately causing cell death. Induction of apoptosis is related to a decrease in membrane fluidity which activates the receptor molecules responsible for apoptosis. ${ }^{52}$ Apoptosis is also inducible by membrane rigidification via lipid peroxidation associated with reactive oxygen species generation $^{53}$ and through the interaction of extracellular $\mathrm{Ca}^{2+}$ with membrane phosphatidylserine. ${ }^{54}$ Antiproliferative agents that modify membrane fluidity have been known to induce apoptosis. ${ }^{55}$ Flavonoids like quercetin can arrest the cell cycle in the $G_{0} / G_{1}$ phase, in the $G_{2} / M$ phase, or in the $S$ phase. ${ }^{47}$ In membrane dynamics, the cell cycle is accompanied by 
membrane fluidity changes, and the membranes of resting cells are more rigid than those of proliferating ones. ${ }^{29}$ Membranerigidifying flavonoids would be effective in preventing changes in the fluidity of tumor cells.

Red wine contains substantial quantities of (-)-epigallocatechin gallate, quercetin, and cyanidin. ${ }^{9}$ Their antiproliferative properties contribute to cancer prevention by blocking cell hyperproliferation. However, the question remains as to whether red wine flavonoids exert such effects in humans because they may be poorly absorbed, highly metabolized, or rapidly excreted. Pharmacokinetic and intervention studies have shown that flavonoid concentrations in plasma after administration may be high enough to show the intrinsic bioactivity of the flavonoids. ${ }^{9,56,57}$ Although aglycones can be absorbed from the small intestine, almost all of the flavonoids, with the exception of flavanols, are present as glycosides in red wine. Flavonoid glycosides are subject to deglycosidation by intestinal bacteria, with subsequent absorption. Because cells in the small intestine exhibit glucoside-hydrolyzing activity, the small intestine possibly acts as an absorption site for flavonoids. ${ }^{58}$ It is of much interest for quercetin that moderate alcohol intake promotes the absorption of quercetin in rat intestines, ${ }^{59}$ which is advantageous, as it allows red wine to display the potential health benefits of flavonoid components.

\section{Conclusion}

This study has identified a possible mechanism for the antiproliferative effects of the flavonoid components of red wine. Flavonoids interact with liposomal and cellular membranes in a structure-dependent manner to decrease their fluidity by preferentially acting at the deeper hydrophobic regions of the lipid bilayers. Cyanidin, quercetin, and (-)-epigallocatechin gallate inhibit the proliferation of tumor cells, together with rigidifying cell membranes. These flavonoids are responsible for the functionality of red wine through their interaction with biomembranes.

\section{Acknowledgment}

This study was supported by a grant from the San-Ei Gen Foundation for Food Chemical Research.

\section{Disclosure}

The author reports no conflicts of interest in this work.

\section{References}

1. Lippi G, Franchini M, Guidi GC. Red wine and cardiovascular health: The "French paradox" revisited. Int J Wine Res. 2010;2:1-7.

2. Ruf JC. Alcohol, wine and platelet function. Biol Res. 2004;37: 209-215.
3. Lagrue-Lak-Hal AH, Andriantsitohaina R. Red wine and cardiovascular risks. Arch Mal Coeur Vaiss. 2006;99:1230-1235. French.

4. Vidavalur R, Otani H, Singal PK, Maulik N. Significance of wine and resveratrol in cardiovascular disease: French paradox revisited. Exp Clin Cardiol. 2006;11:217-225.

5. Opie LH, Lecour S. The red wine hypothesis: From concepts to protective signalling molecules. Eur Heart J. 2007;28:1683-1693.

6. Das S, Santani DD, Dhalla NS. Experimental evidence for the cardioprotective effects of red wine. Exp Clin Cardiol. 2007;12: $5-10$.

7. Waterhouse AL. Wine phenolics. Ann N Y Acad Sci. 2002;957: 21-36.

8. Sandhu AK, Gu L. Antioxidant capacity, phenolic content, and profiling of phenolic compounds in the seeds, skin, and pulp of Vitis rotundifolia (muscadine grapes) as determined by HPLC-DAD-ESI-MS(n). J Agric Food Chem. 2010;58:4681-4692.

9. Manach C, Scalbert A, Morand C, Rémésy C, Jiménez L. Polyphenols: Food sources and bioavailability. Am J Clin Nutr. 2004;79:727-747.

10. Middleton E Jr, Kandaswami C, Theoharides TC. The effects of plant flavonoids on mammalian cells: Implications for inflammation, heart disease, and cancer. Pharmacol Rev. 2000;52:673-751.

11. Di Carlo G, Mascolo N, Izzo AA, Capasso F. Flavonoids: Old and new aspects of a class of natural therapeutic drugs. Life Sci. 1999;65: 337-353.

12. Briviba K, Pan L, Rechkemmer G. Red wine polyphenols inhibit the growth of colon carcinoma cells and modulate the activation pattern of mitogen-activated protein kinases. J Nutr. 2002;132:2814-2818.

13. Oteiza PI, Erlejman AG, Verstraeten SV, Keen CL, Fraga CG. Flavonoidmembrane interactions: A protective role of flavonoids at the membrane surface? Clin Dev Immunol. 2005;12:19-25.

14. Tsuchiya H, Tanaka T, Nagayama M. Antiproliferative effects associated with membrane lipid interaction of green tea catechins. J Health Sci. 2008;54:576-580.

15. Arora A, Byrem TM, Nair MG, Strasburg GM. Modulation of liposomal membrane fluidity by flavonoids and isoflavonoids. Arch Biochem Biophys. 2000;373:102-109.

16. Tsuchiya H. Stereospecificity in membrane effects of catechins. Chem Biol Interact. 2001;134:41-54.

17. Ollila F, Halling K, Vuorela P, Vuorela H, Slotte JP. Characterization of flavonoid-biomembrane interactions. Arch Biochem Biophys. 2002;399: 103-108.

18. Erlejman AG, Verstraeten SV, Fraga CG, Oteiza PI. The interaction of flavonoids with membranes: Potential determinant of flavonoid antioxidant effects. Free Radic Res. 2004;38:1311-1320.

19. Hendrich AB. Flavonoid-membrane interactions: Possible consequences for biological effects of some polyphenolic compounds. Acta Pharmacol Sin. 2006;27:27-40.

20. Tsuchiya H, Nagayama M. Garlic allyl derivatives interact with membrane lipids to modify the membrane fluidity. J Biomed Sci. 2008; 15:653-660.

21. Tsuchiya H. Structure-dependent membrane interaction of flavonoids associated with their bioactivity. Food Chem. 2010;120:1089-1096.

22. Makris DP, Rossiter JT. Heat-induced, metal-catalyzed oxidative degradation of quercetin and rutin (quercetin 3-O-rhamnosylglucoside) in aqueous model systems. J Agric Food Chem. 2000;48:3830-3838.

23. Fernandez MT, Mira ML, Florêncio MH, Jennings KR. Iron and copper chelation by flavonoids: An electrospray mass spectrometry study. J Inorg Biochem. 2002;92:105-111.

24. Tsuchiya H, Ueno T, Mizogami M, Takakura K. Local anesthetics structure-dependently interact with anionic phospholipid membranes to modify the fluidity. Chem Biol Interact. 2010;183:19-24.

25. Tilley L, Thulborn KR, Sawyer WH. An assessment of the fluidity gradient of the lipid bilayer as determined by a set of $n$-(9-anthroyloxy) fatty acids $(n=2,6,9,12,16)$. J Biol Chem. 1979;254:2592-2594.

26. Schoefer L, Braune A, Blaut M. A fluorescence quenching test for the detection of flavonoid transformation. FEMS Microbiol Lett. 2001;204: $277-280$. 
27. Hendrich AB, Malon R, Pola A, Shirataki Y, Motohashi N, Michalak K. Differential interaction of Sophora isoflavonoids with lipid bilayers. Eur J Pharm Sci. 2002;16:201-208.

28. Heim KE, Tagliaferro AR, Bobilya DJ. Flavonoid antioxidants: Chemistry, metabolism and structure-activity relationships. $J$ Nutr Biochem. 2002;13:572-584.

29. Muller CP, Krueger GRF. Modulation of membrane proteins by vertical phase separation and membrane fluidity. Basis for a new approach to tumor immunotherapy. Anticancer Res. 1986;6:1181-1193.

30. Jaruga E, Sokal A, Chrul S, Bartosz G. Apoptosis-independent alterations in membrane dynamics induced by curcumin. Exp Cell Res. 1998;245:303-312.

31. Zhou Y, Hancock JF, Lichtenberger LM. The nonsteroidal anti-inflammatory drug indomethacin induces heterogeneity in lipid membranes: Potential implication for its diverse biological action. PLoS One. 2010;5:e8811.

32. Gaber MH, Ghannam MM, Ali SA, Khalil WA. Interaction of doxorubicin with phospholipid monolayer and liposomes. Biophys Chem. 1998; 70:223-229.

33. Tarahovsky YS, Muzafarov EN, Kim YA. Rafts making and rafts braking: How plant flavonoids may control membrane heterogeneity. Mol Cell Biochem. 2008;314:65-71.

34. Kong JM, Chia LS, Goh NK, Chia TF, Brouillard R. Analysis and biological activities of anthocyanins. Phytochemistry. 2003;64: 923-933.

35. Arts IC, van De Putte B, Hollman PC. Catechin contents of foods commonly consumed in The Netherlands. 2. Tea, wine, fruit juices, and chocolate milk. J Agric Food Chem. 2000;48:1752-1757.

36. Shih PH, Yeh CT, Yen GC. Effects of anthocyanidin on the inhibition of proliferation and induction of apoptosis in human gastric adenocarcinoma cells. Food Chem Toxicol. 2005;43:1557-1566.

37. Hirooka K, Fujita Y. Excess production of Bacillus subtilis quercetin 2,3-dioxygenase affects cell viability in the presence of quercetin. Biosci Biotechnol Biochem. 2010;74:1030-1038.

38. Oak MH, Bedoui JE, Madeira SVF, Chalupsky K, Schini-Kerth VB. Delphinidin and cyanidin inhibit PDGF $_{\mathrm{AB}}$-induced VEGF release in vascular smooth muscle cells by preventing activation of $\mathrm{p} 38$ MAPK and JNK. Br J Pharmacol. 2006;149:283-290.

39. Meiers S, Kemény M, Weyand U, Gastpar R, von Angerer E, Marko D. The anthocyanidins cyanidin and delphinidin are potent inhibitors of the epidermal growth-factor receptor. J Agric Food Chem. 2001; 49:958-962.

40. Montoro P, Braca A, Pizza C, De Tommasi N. Structure-antioxidant activity relationships of flavonoids isolated from different plant species. Food Chem. 2005;92:349-355.

41. Tammela P, Laitinen L, Galkin A, et al. Permeability characteristics and membrane fluidity of flavonoids and alkyl gallates in Caco-2 cells and in phospholipid vesicles. Arch Biochem Biophys. 2004;425:193-199.

42. Rezk BM, Haenen GR, van der Vijgh WJ, Bast A. The antioxidant activity of phloretin: The disclosure of a new antioxidant pharmacophore in flavonoids. Biochem Biophys Res Commun. 2002;295:9-13.

43. Kern M, Fridrich D, Reichert J, et al. Limited stability in cell culture medium and hydrogen peroxide formation affect the growth inhibitory properties of delphinidin and its degradation product gallic acid. Mol Nutr Food Res. 2007;51:1163-1172.
44. Shinitzky M. Membrane fluidity in malignancy. Adversative and recuperative. Biochim Biophys Acta. 1984;738:251-261.

45. Kim HP, Mani I, Iversen L, Ziboh VA. Effects of naturally-occurring flavonoids and biflavonoids on epidermal cyclooxygenase and lipoxygenase from guinea-pigs. Prostaglandins Leukot Essent Fatty Acids. 1998;58:17-24.

46. Seeram NP, Zhang Y, Nair MG. Inhibition of proliferation of human cancer cells and cyclooxygenase enzymes by anthocyanidins and catechins. Nutr Cancer. 2003;46:101-106.

47. Mertens-Talcott SU, Talcott ST, Percival SS. Low concentrations of quercetin and ellagic acid synergistically influence proliferation, cytotoxicity and apoptosis in MOLT-4 human leukemia cells. Nutr Cancer. 2003;133:2669-2674.

48. Daoud SS. Cell membranes as targets for anti-cancer drug action. Anticancer Drugs. 1992;3:443-453.

49. Mestre JR, Chan G, Zhang F, et al. Inhibition of cyclooxygenase-2 expression. An approach to preventing head and neck cancer. Ann NY Acad Sci. 1999;889:62-71.

50. Hou DX, Yanagita T, Uto T, Masuzaki S, Fujii M. Anthocyanidins inhibit cyclooxygenase-2 expression in LPS-evoked macrophages: Structure-activity relationship and molecular mechanisms involved. Biochem Pharmacol. 2005;70:417-425.

51. Czapla K, Korchowiec B, Rogalska E. Differentiating oxicam nonsteroidal anti-inflammatory drugs in phosphoglyceride monolayers. Langmuir. 2010;26:3485-3492.

52. Yeruva L, Elegbede JA, Carper SW. Methyl jasmonate decreases membrane fluidity and induces apoptosis via tumor necrosis factor receptor 1 in breast cancer cells. Anticancer Drugs. 2008;19: 766-776.

53. Chang H, Lin H, Yi L, et al. 3,6-Dihydroxyflavone induces apoptosis in leukemia HL-60 cells via reactive oxygen species-mediated p38 MAPK/JNK pathway. Eur J Pharmacol. 2010;648:31-38.

54. Goto K, Tatsumi K, Nagata T, Komizu Y, Ueoka R. Specific interaction between $\mathrm{Ca}^{2+}$ and anionic lipids in cancer cells along with apoptosis. Biol Pharm Bull. 2011;34:439-442.

55. Mandlekar S, Kong AN. Mechanisms of tamoxifen-induced apoptosis. Apoptosis. 2001;6:469-477.

56. Chow HHS, Cai Y, Alberts DS, et al. Phase I pharmacokinetic study of tea polyphenols following single-dose administration of epigallocatechin gallate and polyphenon E. Cancer Epidemiol Biomarkers Prev. 2001;10:53-58.

57. Goldberg DM, Yan J, Soleas GJ. Absorption of three wine-related polyphenols in three different matrices by healthy subjects. Clin Biochem. 2003;36:79-87.

58. Murota K, Terao J. Antioxidative flavonoid quercetin: Implication of its intestinal absorption and metabolism. Arch Biochem Biophys. 2003; 417:12-17.

59. Dragoni S, Gee J, Bennett R, Valoti M, Sgaragli G. Red wine alcohol promotes quercetin absorption and directs its metabolism towards isohamnetin and tamarixetin in rat intestine in vitro. Br J Pharmacol. 2006;147:765-771.
International Journal of Wine Research

\section{Publish your work in this journal}

The International Journal of Wine Research is an international, peer-reviewed open-access, online journal focusing on all scientific aspects of wine, including: vine growing; wine elaboration; human interaction with wine; and health aspects of wine. The journal provides an open access platform for the reporting

\section{Dovepress}

of evidence based studies on these topics. The manuscript management system is completely online and includes a very quick and fair peer-review system, which is all easy to use. Visit http://www.dovepress.com/testimonials.php to read real quotes from some of our published authors. 